\title{
Effects of Ultrasound on Behavior and Dopamine Content in Striatum of Parkinson's Disease Model Mouse
}

\author{
Weiwei Wang ${ }^{a}$, Li Li ${ }^{b}$, Wei Wu ${ }^{c, *}$, Wanlin Zhang ${ }^{d}$, Ying Gao ${ }^{e}$ and Chen Chen ${ }^{f}$ \\ School of Southeast University, Jiangsu 210009, China; \\ a1715556404@qq.com, b1404157708@qq.com, c101005800@seu.com, d1292665976@qq.com, \\ e3236027533@qq.com, f915666024@qq.com
}

Keywords: ultrasound, PD, behavior, DA.

\begin{abstract}
In this research, we established a MPTP mouse model of PD, then ultrasound was used to stimulate the model to explore the effects of ultrasound on behavior and dopamine content in striatum. With the prolongation of ultrasonic stimulation time, the delay of observation time, the amount of DA in the striatum of the ultrasonic treatment group was increasing. Using ultrasound with a frequency of $1 \mathrm{MHz}$ at an intensity of $0.3 \mathrm{~W} / \mathrm{cm}^{2}$ over $15 \mathrm{~min}$ for $1 \mathrm{~d}, 5 \mathrm{~d}$ or $10 \mathrm{~d}$ respectively, in each group, there was a significant difference in the content of DA in the striatum compare to PD observation group at the same observation time $(P<0.05)$. Especially, the DA content in the striatum of the mice treated with the ultrasound for $10 \mathrm{~d}$ was not statistically significant compared with the control group $(P>0.05)$. Combined with the results of climbing test and open field test and general behavioral changes, the difference between ultrasound group and PD group reached a significant level $(P<0.05)$. In summary, ultrasound can increase the DA content in the brain and improve the motor capacity of PD model. It suggests that ultrasound may be a safe and effective new method of PD treatment.
\end{abstract}

\section{Introduction}

Parkinson's disease (PD) is a common neurodegenerative disorder characterized by the reduce of the striatal DA level, which is related to the degeneration, death and deletion of dopaminergic neurons in the substantia nigra pars compacta $(\mathrm{SNc})$, as well as the decrease of dopamine biosynthesis ability of the surviving neurons [1-3]. A series of clinical symptoms including resting tremor, rigidity, bradykinesia and postural instability will appear when DA content is lower than $70 \%$. The gold standard for the treatment of PD is Levodopa therapy, which is aimed at increasing the availability of striatal dopamine and typically result in a brisk symptomatic improvement in most PD patients. Unfortunately, this response becomes less reliable and less predictable over time, medication related complications such as dyskinesia and "on-off" fluctuations develop in a majority of patients [4]. Because of the side effect of Levodopa therapy, researchers started to explore new therapies for PD.

Ultrasound, as a new physical therapy, has been shown to be an effective neuronal activity regulator. William et al [5] experiments found that ultrasound on the cat's lateral geniculate body can inhibit the visual cortex evoked potential. Bauer et al [6] in the study results confirmed that after the end of ultrasound intervention in patients with tremor ease, six months after treatment and the Webster score was significantly improved. Wang et al found that GDNF levels were increased in the striatum of PD rats after lipid-coated GDNF microspheres administration following low frequency ultrasound stimulation $(20 \mathrm{kHz}, 5 \mathrm{~min}$ per day, daily for 4 weeks). Moreover, GDNF microspheres reduced apomorphine-induced rotations, and increased striatal dopamine and nigral tyrosine hydroxylase (TH) levels in PD rats. In this research, we established a MPTP mouse model of PD, then using ultrasound with a frequency of $1 \mathrm{MHz}$ at an intensity of $0.3 \mathrm{~W} / \mathrm{cm}^{2}$ stimulate the head of mouse to explore the effects of ultrasound on Behavior and Dopamine Content in striatum. 


\section{Materials and methods}

\subsection{Animals.}

Sixty six rats(C57/BL6, males, 20-22 g, 6-8 weeks old) were used in this study. All animals were purchased from Nanjing University-Nanjing Biomedical Research Center. According to previous experimental results, the animal model of PD received i.p. injection of $30 \mathrm{mg} / \mathrm{kg} / \mathrm{d}$ MPTP (Sigma) and continued for 5 days. All the animals were divided into ultrasonic stimulation group (US1-9), the PD observation group (PD) and the control group of six.

\subsection{Ultrasonic radiation parameters and radiation methods.}

As previously reported $[7,8]$, the ultrasonic radiation frequency $f$ was chosen to be $1 \mathrm{MHz}$, and the ultrasonic radiation frequency was measured with an oscilloscope with a frequency meter (TBS1102, Tektronix) before the ultrasonic radiation. The error range was $\leq \pm 1 \%$ Time $(\mathrm{t})$ is set to $5 \mathrm{~min}, 10 \mathrm{~min}, 15 \mathrm{~min}$; ultrasonic radiation days (D) is set to $1 \mathrm{~d}, 5 \mathrm{~d}, 10 \mathrm{~d}$. Combined with FDA [9-10] recommended ultrasonic sound intensity shall not exceed $0.3 \mathrm{~W} / \mathrm{cm}^{2}$, the final ultrasonic radiation power is set to $0.3 \mathrm{~W} / \mathrm{cm}^{2}$, the error range of $\leqslant \pm 10 \%$, each ultrasound before the use with ultrasonic power meter (Model UPM-DF-1E, Ohmic) to calibrate to ensure the accuracy and consistency of ultrasonic radiation power.

After the MPTP model of PD established, using the ultrasound stimulate the head of mouse of US group which was shaved the hair coated with ultrasound coupling gel.

\subsection{Evaluation of athletic ability.}

\subsubsection{General behavior.}

After treated the mice with MPTP for 5 days, whether the animals showed a series of symptoms which was similar to PD, including in autonomic activity, tremor, hind limb stiffness, vertical tail, vertical hair, curled up and et.al

\subsubsection{Pole climbing test.}

A ball with a diameter of $2.5 \mathrm{~cm}$ was fixed at the top of a vertical rod with a length of $50 \mathrm{~cm}$ and a diameter of $1 \mathrm{~cm}$. The whole device was wrapped with gauze to increase the grip. During the test, the bottom of the device was placed in a rat cage filled with mouse litter increase the adaptability of mice. Place the mouse head up on the ball and record the T-turn and the total time (movement time, T-total) consumed by the limbs on the table. If the mouse falls from the climbing device or the upward crawling occurs during the descending of the mouse, it is not recorded. Before the start of the experiment, mice were trained for climbing for 3 days and recorded data on the fourth day. Each mouse was continuously measured 5 times.

\subsubsection{Open field test.}

According to the operation procedure, the open field test device program (JLbehv-L, Shanghai Jigong Software Technology Co., Ltd) was set up. The bottom of the open field was divided into 16 small cells. The mice were placed in the middle lattice of the test area while taking pictures and timing. The mice were observed and recorded 3 minutes. The exercise distance (s), mean velocity (v) and the number of lattice (n) were measured to measure the activity of mice. During the course of the experiment, keep the test environment quiet and avoid disturbing the mice. At the same time, it is necessary to avoid mutual interference between mice. After the end of each test, the inner wall of the open field device is wiped with alcohol cotton balls to eliminate the residual stool, urine and smell of the last test mice.

\subsubsection{Determination of DA in striatum.}

Mice were anesthetized with 10\% chloral hydrate (Chengdu Kelon Chemical Reagent Factory) at a dose of $0.1 \mathrm{~mL} / 20 \mathrm{~g}$ body weight and sacrificed and their brains were quickly removed and placed on ice. Striatum was found and placed in the numbered EP tube weighing and record. The tissue was placed in a glass grinder, and $400 \mu \mathrm{L}$ of $5 \%$ perchloric acid (Shanghai Golden Deer Chemical Reagent Factory) was added to each tissue, centrifuged at $15,000 \mathrm{r} / \mathrm{min}$ for $20 \mathrm{~min}$ at $4^{\circ} \mathrm{C}$. Under the same condition, centrifuged once again and the supernatants were taken for measurements of DA by HPLC (Agilent1200, Agilent). 


\subsection{Statistical analysis.}

All the experimental results were created in Excel and analyzed with SPSS19.0. Data are expressed as mean \pm SEM. Statistical analysis was conducted by one-way analysis of variance (ANOVA). Inspection level $\alpha=0.05$ (bilateral).

\section{Results}

\subsection{General behavior changes.}

After the first injection of MPTP about 10min, the mice showed vertical hair, vertical tail, limb tremor, arch back gates, behavioral activity slow, gait instability, curling and other behavior changes, after 24 hours the symptoms has disappeared. However, with the increase of the number of injection, the effect of drug accumulation on the behavior was gradually obvious and the behavior changes including in movement of slowed down, limb stiffness, gait instability, low response was significant. At the end of the modeling, the behavioral changes of the model mice in the PD model group were continuous, and there was a slight improvement as the extension of time, however, there is a difference when compared with the control group and the US group.

\subsection{Pole climbing test.}

In the PD observation group, the T-turn and T-total of the model mice decreased gradually with the prolongation of the observation time. And the T-turn of the $1 \mathrm{~d}, 5 \mathrm{~d}$ and $10 \mathrm{~d}$ groups were $(3.877 \pm$ $0.641 \mathrm{~s} ; 2.182 \pm 0.327 \mathrm{~s} ; 1.581 \pm 0.282 \mathrm{~s})$, T-total were $(25.246 \pm 2.264 \mathrm{~s} ; 13.836 \pm 2.205 \mathrm{~s} ; 12.870 \pm 1.757 \mathrm{~s})$. And the difference between the control group $(\mathrm{T}$-turn $=0.557 \pm 0.094 \mathrm{~s}$; T-total $=5.369 \pm 0.471 \mathrm{~s})$ reached a significant level $(\mathrm{p}<0.05)$.

In the US group, T-turn and T-total showed a decreasing trend with the prolongation of ultrasonic radiation time and the days of ultrasonic radiation. There was no significant difference between the US10d group and the control group ( $>0.05)$, indicating that the exercise ability of US10 group had returned to normal level. T-turn and T-total of mice of US group were significantly improved compared with PD observation group, as shown in Fig. 1. Ultrasonography could improve the ability of climbing of MPTP mice and improve their dyskinesia.

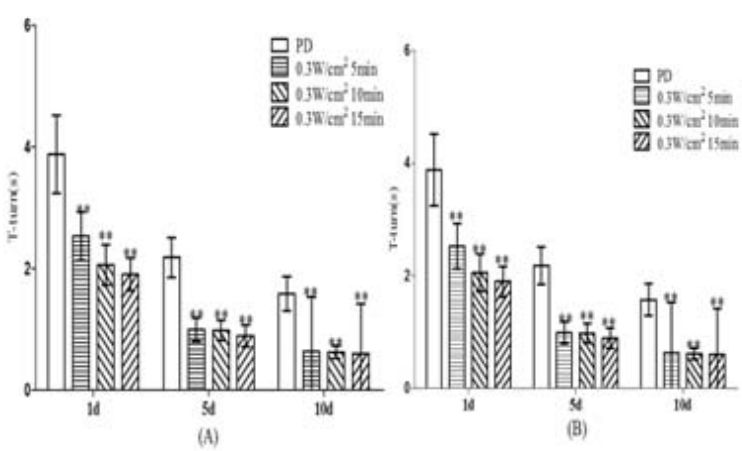

Fig.1 The Pole climbing test of Ultrasound treatment group and PD model group $(N=6$ ). ( A for the T-turn; B for the T-total. *p $<0.05$, $* * \mathrm{p}<0.01$ )

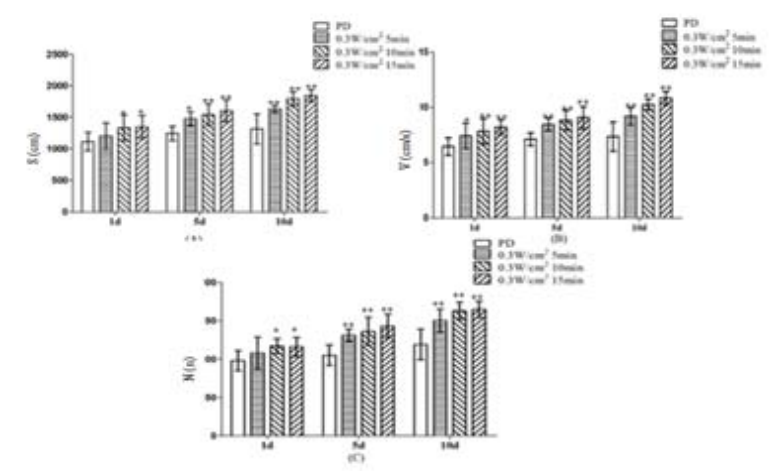

Fig.2 The open field test of Ultrasound treatment group and PD model group $(N=6$ ). (A for the Distance(s); B for the mean velocity(v) and the $\mathrm{C}$ for the number of lattice(n) $. * p<0.05, * * p<0.01)$

\subsection{Open field test.}

In the PD model observation group, the test results of the model mice were gradually improved with the observation time prolonged. The exercise distance (s), mean velocity (v) and the number of lattice (n) of the PD1d, PD5d, PD10d model observation group were $(1115.199 \pm 142.862 \mathrm{~cm}$; $1244.808 \pm 111.562 \mathrm{~cm} ; 1317.254 \pm 236.675 \mathrm{~cm}),(6.458 \pm 0.807 \mathrm{~cm} / \mathrm{s} ; 7.121 \pm 0.589 \mathrm{~cm} / \mathrm{s} ; 7.354 \pm 1.314 \mathrm{~cm} / \mathrm{s}$ ),$(98.333 \pm 13.033$ cells; $105.667 \pm 13.441$ cells; $119.000 \pm 19.940$ cells $)$ respectively, and had significant difference between the control group $(\mathrm{s}=1883.805 \pm 44.823 \mathrm{~cm} ; \mathrm{v}=11.050 \pm 0.570 \mathrm{~cm} / \mathrm{s}$; $\mathrm{n}=167.167 \pm 10.108$ cells $)$ respectively $(\mathrm{p}<0.05)$. In the US group, except for 


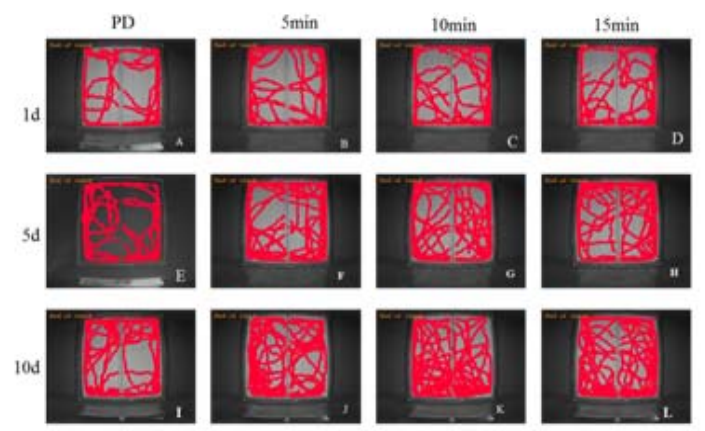

Fig.3 The map of open field test of Ultrasound treatment group and PD model group $(\mathrm{N}=6)$.

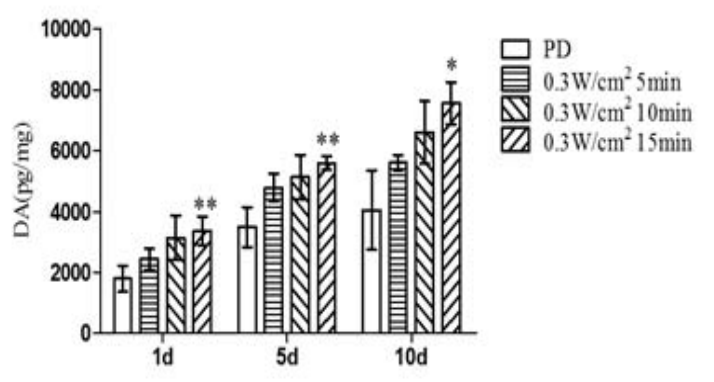

Fig.4 The DA content of Ultrasound treatment group and PD model group $(\mathrm{N}=6) .\left({ }^{*} \mathrm{p}<0.05,{ }^{* *} \mathrm{p}<0.01\right)$

group $1\left(f=1 \mathrm{MHz}, P=0.3 \mathrm{~W} / \mathrm{cm}^{2}, t=5 \mathrm{~min}, D=1 \mathrm{~d}\right)$, others were compared with the PD model observation group of the corresponding time in the exercise distance (s), mean velocity (v) and the number of lattice (n) and the difference reached a significant level, as shown in Fig. 2. There was no significant difference between the group $9\left(f=1 \mathrm{MHz}, P=0.3 \mathrm{~W} / \mathrm{cm}^{2}, t=15 \mathrm{~min}, D=10 \mathrm{~d}\right)$ and control group ( $p>0.05)$. According to the open field test results of the model mice showed that the ultrasonic radiation could restore the open field test ability of the model mice and improve the motor function.

\subsection{Determination of DA in striatum.}

The DA contents in the striatum of PD1d, PD5d and PD10d group were $(1789.292 \pm 424.532 \mathrm{pg} / \mathrm{mg}$; $3494.452 \pm 654.031 \mathrm{pg} / \mathrm{mg} ; 4049.445 \pm 1302.687 \mathrm{pg} / \mathrm{mg})$. Compared with control group respectively, the difference had a significant level $(\mathrm{P}<0.05)$.

In the US group, with the prolongation of the ultrasonic radiation time and days of ultrasonic radiation, the DA content of the US model mice increased. The contents of DA were $(3364.942 \pm 460.210 \mathrm{pg} / \mathrm{mg} ; 5590.260 \pm 228.044 \mathrm{pg} / \mathrm{mg} ; 7560.983 \pm 692.088 \mathrm{pg} / \mathrm{mg})$ after $1 \mathrm{~d}, 5 \mathrm{~d}$ and $10 \mathrm{~d}$ treatment with ultrasonic irradiation at the frequency of $1 \mathrm{MHz}$ and power of $0.3 \mathrm{~W} / \mathrm{cm}^{2}$ for $15 \mathrm{~min}$. There was significant different between the treatment group and the PD group of corresponding time $(\mathrm{P}<0.05)$, as shown in Fig. 2. In addition, there had no significant difference between control group and treatment group when the mice were irradiated with $f=1 \mathrm{MHz}, \mathrm{P}=0.3 \mathrm{~W} / \mathrm{cm}^{2}, \mathrm{t}=15 \mathrm{~min}$ and $\mathrm{D}$ $=10 \mathrm{~d}$ in $\mathrm{DA}$ content $(\mathrm{P}>0.05)$.

\section{Discussion}

At present, the nerve stimulation method has been widely used in the study of nervous system and mental illness. Common treatment methods about nerve stimulation methods include electromagnetic-, optical- and acoustic- mediated [11-14]. The electrical stimulation method is to improve the movement disorder by blocking the abnormal discharge of the nucleus of the brain lesions. Today, the application of more brain deep electrical stimulation can stimulate the brain deep region [15], but the electrode placement surgery is a method of high degree invasion. Emerging transcranial DC stimulation avoids the drawbacks of intrusiveness, but there are still shortcomings in the precise positioning of the deep region [16, 17]. Magnetic stimulation is a bio-stimulating technique that utilizes the magnetic field to act on the cerebral cortex to produce an induced electric field, which produces the action potential of the cortical neurons that changes the induced current, thereby affecting the material metabolism and neural activity in the brain. Magnetic stimulation technology achieves noninvasive possibilities, but there are limitations of parameter measurement imbalance and technical feasibility of focusing on the area of action. On the other hand, the magnetic field spatial resolution limits feasibility when it in the deep brain stimulation [18]. Ultrasound, as a safe, noninvasive, spatial resolution and noninvasive means, is increasingly recognized for disease diagnosis and treatment. Ultrasound can penetrate the human body and brain tissue in a noninvasive manner to focus on the area in millimeters, combined with its role in regulating neuronal activity, showing its application in neuroscience. About 80 years ago, by regulating the neuromuscular activity of frogs and turtles, ultrasound has been shown to be an effective neuromodulation tool [19]. And 
recent studies have confirmed the transcranial focus ultrasound on the human primary body sensory cortex regulation [20]. In a study by Bauer et al. [6], focused ultrasound was used to intervene in patients with idiopathic PD syndrome who had a major clinical symptom of resting tremor using magnetic resonance imaging system. The results showed that the degree of tremor was relieved after the end of the intervention and after six months treatment, the score was significantly higher, however, ultrasound microfocus ultrasound, which is used in this study, requires guidance from external instruments. The aim of our study was to use the ultrasound in the safety limits to radiate the whole brain without using external instruments to increase the DA content in the striatum improve the symptoms of the Parkinson's Disease.

The main pathological features of PD is the decrease of DA content in the brain, the degree of decrease in DA content in the basal ganglia is related to the degeneration and deletion of DA-neurons in the substantia nigra-striatum, and the decrease of DA biosynthesis in the residual neuronal cells. In this study, we found that the release of DA in striatum of US group model gradually increased with the prolongation of ultrasonic radiation time and radiation days, using the ultrasound with a the frequency of $1 \mathrm{MHz}$ at an intensity of $0.3 \mathrm{~W} / \mathrm{cm}^{2}$ stimulate the MPTP model mice. And combined with the results of climbing test and open field test and general behavioral changes, there had a significant difference between control group $(P>0.05)$, suggesting that the ultrasonic radiation has a certain effect on the motor behavior of MPTP mice. DA is composed of substantia nigra neurons, which is transported by the substantia nigra - striatum fibers to the DA nerve endings and transported through the vesicles of the type II vesicle monoamine transporter and stored in the vesicles of the DA nerve endings. When nerve impulses are passed, DA is released into the synaptic cleft and binds to the postsynaptic membrane DA receptor to play a physiological effect $[8,20]$. It can be seen that the decrease of DA in the pathogenesis of PD may be related to the number of TH-positive neurons in the substantia nigra of the midbrain, and may also be related to the integrity of the nerve fibers on the DA pathway. In this study, the increase in DA content and the symptoms of the model mice were improved by ultrasound irradiation. However, the mechanism of promoting the release of DA by ultrasonic radiation should be discussed in the future

\section{Conclusion}

1 Ultrasound radiation can promote the increase of DA content in striatum of MPTP model mice;

2 Ultrasound irradiation of MPTP model mice with frequency of $1 \mathrm{MHz}$ and power of $0.3 \mathrm{~W} / \mathrm{cm}^{2}$ showed a significant improvement in the behavioral indexes, including in general behavior, pole climbing test and open field test, after 10 days of continuous irradiation for $15 \mathrm{~min}$. and compared with the control group, there was no statistical differences.

\section{References}

[1]. Hess C W, Okum M S. Diagnosing Parkinson Disease. Movement Disorder, 2016, 22(4): 1047 1063.

[2]. Katzenschlager R. Parkinson's Disease: Recent Advance. Journal of Neurology, 2014, 261(5): 1031-1036

[3]. Todorova A, Jenner P, Ray Chaudhuri K. Non-Motor Parkinson's: Integral to Motor Parkinson's, Yet Often Neglected. Practical Neurology, 2014, 14(5): 310-322.

[4]. Schapira AHV. Present and future drug treatment for Parkinson's disease [J]. J Neurol Neurosurg Psychiatry, 2005, 76: 1472-1478.

[5]. Fry F. J., Ades H. W., Fry W. J. Production of Reversible Changes in the Central Nervous System by Ultrasound. Science, New Series.1958.127:3289.

[6]. Bauer R, Martin E, Haegele-Link S, et al. Noninvasive Functional Neurosurgery Using Transcranial MR Imaging-Guided Focused Ultrasound. Parkinsonism Related Disorder, 2014, 20(1):S197-S199.

[7]. D'Souza A L, Tseng J R, Pauly K B, et al. A Strategy for Blood Biomarker Amplification and Localization Using Ultrasound. Proc Natl Acad Sci U S A, 2009, 106(40): 17152-17157. 
[8]. Peng D, Xu T, Mason T J, et al. A Study of Ovarian Cancer Biomarker Amplification Using Ultrasound for Early Stage Detection. Ultrasonics, 2014, 54(2): 451-454.

[9]. U.S. Department of Health and Human Services, Food and drug administration center for devices and radiological health:information for manufacturers seeking market clearance of diagnostic ultrasound systems, $<$ https: //www.fda.gov/downloads/Medical Devices/Device Regulation and Guidance/ Guidance Documents/ ucm070911.pdf $>$.

[10]. Natalie Phipps, Acoustic intensity measurement system: application in localized drug delivery. San Jose: San Jose State University, 2010.

[11]. Hallett M.Transcranial Magnetic Stimulation: A Primer. Neuron, 2007, 55(2): 187-199.

[12]. Ressler K J, Mayberg H S. Targeting Abnormal Neural Circuits in Mood and Anxiety Disorders: From the Laboratory to the Clinic. Nature Neuroscience, 2007, 10(9): 1116-1124.

[13]. Deisseroth K.Optogenetics. Nature Methods, 2010, 8(1): 26-29.

[14]. Li G, Zhao H\& Zhou H, et al. Improved Anatomical Specificity of Non-Invasive NeuroStimulation by High Frequency (5 MHz) Ultrasound. Scientific Reports, 2016, 6(1):1-11.

[15]. Faggiani E, Benazzouz A. Deep Brain Stimulation of the Subthalamic Nucleus in Parkinson'S Disease: From History to the Interaction with the Monoaminergic Systems. Progress in Neurobiology, 2017, 151: 139-156.

[16]. Cambiaghi M, Velikova S, Gonzalez-Rosa J J, et al. Brain Transcranial Direct Current Stimulation Modulates Motor Excitability in Mice. European Journal of Neuroscience, 2010, 31(4): 704-709.

[17]. Brunoni A R, Nitsche M A, Bolognini N, et al. Clinical Research with Transcranial Direct Current Stimulation (tDCS): Challenges and Future Directions. Brain Stimulation, 2012, 5(3): 175-195.

[18]. Bystritsky A, Korb A S, Douglas P K, et al. A Review of Low-Intensity Focused Ultrasound Pulsation. Brain Stimul, 2011, 4(3): 125-136.

[19]. Harvey, E. N. The Effect of high Prequency Sound Waves on Heart Muscle and Other Irritable Tissues.Am. J. Physiol.1929.91:284.

[20]. Panczykowski D M, Monaco E R, Friedlander R M. Transcranial Focused Ultrasound Modulates the Activity of Primary Somatosensory Cortex in Humans . Neurosurgery, 2014, 74(6): N8. 